\title{
Infraestrutura Brasileira para Produção de Madeira Serrada de Reflorestamento
}

\author{
Alexandre Nascimento de Almeida ${ }^{1}$, João Carlos Garzel Leodoro da Silva ${ }^{2}$, \\ Humberto Angelo 3
}

${ }^{1}$ Curso de Gestão Ambiental e do Agronegócio, Faculdade UnB de Planaltina - FUP, Universidade de Brasília - UnB, Planaltina/DF, Brasil

${ }^{2}$ Departamento de Economia Rural e Extensão, Universidade Federal do Paraná- UFPR, Curitiba/PR, Brasil ${ }^{3}$ Departamento de Engenharia Florestal, Faculdade de Tecnologia, Universidade de Brasília - UnB, Brasília/DF, Brasil

\begin{abstract}
RESUMO
Pode-se até discutir o tipo e o grau de interferência do Estado em uma nação, porém é inegável a sua importância no desenvolvimento de qualquer economia, seja como indutor do desenvolvido ou como garantidor das regras e normas em questão. O presente trabalho teve como objetivo analisar a eficiência do Estado brasileiro na criação de um ambiente competitivo para as empresas de madeira serrada de silvicultura. A análise foi fundamentada conforme a percepção dos empresários do segmento, tendo como instrumentais analíticos o gráfico Boxplot e o teste não paramétrico de Mann-Whitney. Os resultados minimizaram problemas em relação à falta de madeira e energia, e distinguiram como pontos críticos a escassez de mão de obra e crédito, bem como a insuficiente condição da infraestrutura para transporte e treinamento de recursos humanos.
\end{abstract}

Palavras-chave: processamento mecânico, economia florestal, competitividade, Pinus spp.

\section{Brazilian Infrastructure for Production of Sawn Wood from Reforestation}

\begin{abstract}
One can even discuss the type and degree of State interference in a nation, but its importance to the development of any economy is undeniable, either as an inducer of development or as a guarantor of the rules and regulations in question. The objective of this study was to analyze the efficiency of the Brazilian State in creating a competitive environment for softwood businesses. The analysis was based on the perceptions of entrepreneurs of this segment using Boxplot graph and nonparametric Mann-Whitney test as analytical tools. The results minimized problems related to the lack of wood and energy, and identified the following as critical points: scarcity of labor and credit, poor transport infrastructure, and training in human resources.
\end{abstract}

Keywords: mechanical processing, forest economy, competitiveness, Pinus spp. 


\section{INTRODUÇÃO}

A competitividade é um dos princípios da economia liberal, que teve como principais precursores David Ricardo e Adam Smith (Andrioli, 2003), sendo que, para Smith, a ideia básica da concorrência é que, uma vez competindo entre si, os atores envolvidos automaticamente estariam contribuindo para o progresso geral da sociedade.

No entanto, o que se observou, na História, é que a livre concorrência eliminou a si mesma, constituindo monopólios e oligopólios privados; ainda, com o advento do neoliberalismo, o Estado passou a ser um instrumento estimulador da competitividade. Veremos, assim, que, com a concorrência matando a concorrência, foi preciso constituir um neoliberalismo para salvar a ideia de liberdade econômica. Enquanto o liberalismo clássico pedia que o Estado não interferisse, para que a concorrência pudesse produzir todos os seus bons efeitos, o neoliberalismo pede ao Estado que se mexa para assegurar que a concorrência possa existir. (Salleroun, 1979).

A questão que se coloca é como o Estado deve apoiar para manter e incrementar essa competitividade. Há quem advogue a favor de uma liberalização acelerada dos mercados, com vistas a prover a maior concorrência, o que, na visão da globalização, representa homogeneidade crescente das estruturas e das relações da modernidade do capitalismo, podendo a aceleração desse processo só trazer benefícios.

Por outro lado, Furtado (1992) adverte que o processo de adesão voluntária e acelerada à globalização, e a concorrência mundial, sem a criação de espaços de acumulação e de desenvolvimento dotados de alguma autonomia e de conteúdo sistêmico, acelera e agrava um processo que é, em si mesmo, dotado de graves consequências negativas para uma nação periférica.

A importância da concorrência como motor para o progresso, em qualquer sociedade, é inegável. Porém, para que ocorra competição, é preciso igualdade de condições, pois, caso contrário, o que ocorrerá é o domínio do lado mais forte e, consequentemente, o desaparecimento do lado mais fraco e da concorrência. Nesse aspecto, destaca-se a importância fundamental do Estado em prover as condições necessárias para que uma determinada indústria concorra em igualdades de condições, garantindo uma concorrência permanente entre as empresas.

Graças às vantagens naturais e aos intensivos esforços em silvicultura, o Brasil consegue produzir a madeira mais barata do mundo (Almeida, 2006). Considerando-se esta vantagem e o fato de que a indústria madeireira não demanda um desenvolvimento tecnológico de Primeiro Mundo, não é preciso muito para tornar o País uma grande referência mundial nesse segmento, como já vem ocorrendo com o de celulose. Ainda segundo o autor, diferentemente do segmento madeireiro, o segmento de celulose, historicamente, tem obtido um forte apoio do Estado.

Apesar do grande potencial, a situação do segmento madeireiro encontra-se bem aquém dos melhores padrões mundiais, predominando empresas com tecnologias obsoletas, pouco especializadas, descapitalizadas e sem escala (Almeida et al., 2011).

O objetivo deste trabalho é analisar a eficiência do Estado na criação de um ambiente competitivo para as empresas madeireiras. Especificamente, avaliaram-se os pontos críticos da estrutura interna para a produção de madeira serrada, conforme a percepção dos empresários do segmento.

\section{METODOLOGIA}

\subsection{Material}

Os dados foram obtidos por meio da aplicação de questionário estruturado direcionado à administração das empresas madeireiras focadas no desdobro de Pinus e Eucalipto, sendo coletados durante o período de janeiro a julho de 2008. A amostragem foi aleatória e totalizou 66 empresas das Regiões Sul e Sudeste, alcançando tamanho suficiente para aplicação dos métodos propostos.

As perguntas do questionário relacionaram-se ao papel do Estado no provimento de uma estrutura adequada para o desenvolvimento do segmento de madeira serrada, buscando captar a percepção do empresário em relação a oito aspectos:

1. A disponibilidade de madeira-DM. 
2. A disponibilidade de mão de obra com habilidades técnicas - MOHT.

3. A disponibilidade de mão de obra com habilidades administrativas - MOHA.

4. A infraestrutura para transportes - IET.

5. A disponibilidade de capital - DC.

6. Disponibilidade de energia - DE.

7. A infraestrutura pública para pesquisa, educação e treinamento doméstico para aspectos tecnológicos - IEAT.

8. A infraestrutura pública para pesquisa, educação e treinamento doméstico para aspectos administrativos - IEAA.

A escala empregada foi a de diferencial semântico (Figura 1), sendo apresentadas as variáveis analisadas e permitindo os entrevistados posicionarem a força de sua opinião em uma escala de sete pontos. Os três primeiros pontos direcionaram para uma situação de inadequação, os três últimos para uma adequação e o valor 4 significou uma posição de indiferença.

\subsection{Métodos}

Os dados foram analisados inicialmente por análise gráfica e, posteriormente, pelo teste não paramétrico de Mann-Whitney. O gráfico utilizado para representar os resultados é do tipo Boxplot, permitindo visualizar a mediana, a dispersão interquartil, as observações máximas e mínimas, e, principalmente, as observações atípicas (outliers) para cada variável (Figura 2).

São considerados outliers todas aquelas observações que estão a mais de 1,5 quartil do extremo da caixa $[3 \times(\mathrm{Q} 3-\mathrm{Q} 1)$ a partir do valor

\section{Significado da caixa de opção}

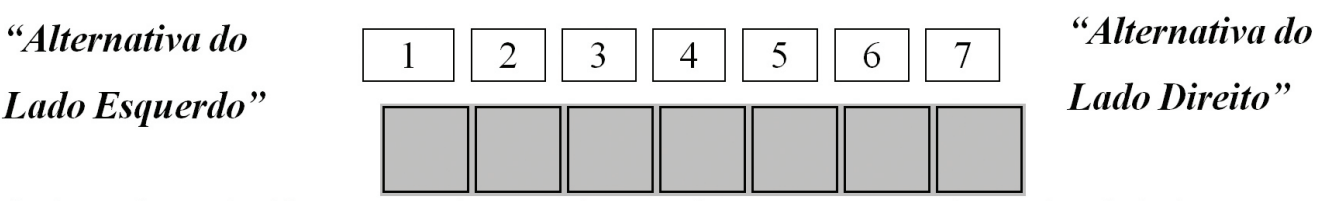

Selecionando 1: significa que você concorda completamente com a alternativa do lado esquerdo. Selecionando 2: significa que você concorda amplamente com a alternativa do lado esquerdo. Selecionando 3: significa que você concorda de certa forma com a alternativa do lado esquerdo. Selecionando 4: significa que você é indiferente às duas alternativas.

Selecionando 5: significa que você concorda de certa forma com a alternativa do lado direito. Selecionando 6: significa que você concorda amplamente com a alternativa do lado direito. Selecionando 7: significa que você concorda completamente com a alternativa do lado direito.

Figura 1. Escala aplicada.

Figure 1. Scale applied.

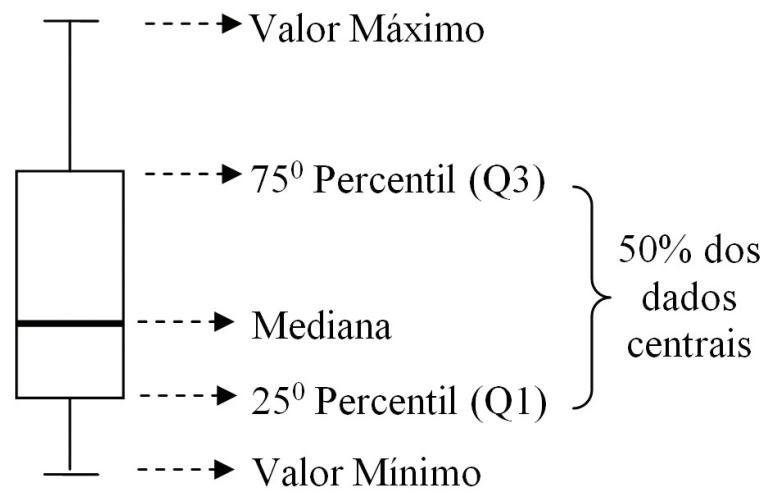

Assimetria à direita: A distância do valor mínimo até Q1 excede em muito a distância do valor máximo até Q3

\section{Assimetria à esquerda: A} distância de Q3 ao valor máximo excede em muito a distância do valor mínimo até Q1

Figura 2. Interpretação do gráfico Boxplot.

Figure 2. Interpretation of the Boxplot chart. 
mínimo ou máximo]. Todas as observações atípicas foram excluídas antes da aplicação do teste de MannWhitney.

Conforme Siegel \& Castellan (2006), o teste de Mann-Whitney é um dos testes não paramétricos mais poderosos, sendo uma alternativa útil para o teste paramétrico $t$ quando o pesquisador deseja evitar as suposições do teste $t$, ou quando a mensuração na pesquisa é mais fraca do que a dada em escala intervalar, ou seja, dados em uma escala ordinal.

O teste de Mann-Whitney foi aplicado para comparar a situação das oito variáveis analisadas, buscando identificar os pontos críticos da infraestrutura para produção de madeira serrada.

Em vez de se basear em parâmetros da distribuição normal, como a média e a variância, o teste de Mann-Whitney baseia-se nas ordenações da variável. Conforme Hoffmann (2006), pode-se descrever o teste de Mann-Whitney nas seguintes etapas:

\section{$1^{a}$ Etapa}

Reuniram-se as duas amostras e atribuiu-se, a cada uma das observações, um número de ordem ou posto, de 1 a N, que corresponde à posição ocupada pela observação em uma sequência ordenada de acordo com o valor crescente das observações. Constatando-se que existem duas ou mais observações com valores iguais, atribuímos a cada uma das amostras a média aritmética dos postos ocupados pelas observações iguais.

\section{$2^{a}$ Etapa}

Após a ordenação dos valores, pode-se obter a estatística do teste de Mann-Whitney pela escolha do menor valor de U, calculado pelas Equações 1 e 2 .

$U_{1}=n_{1} n_{2}+\frac{n_{1}\left(n_{1}+1\right)}{2}-R_{1}$

$U_{2}=n_{1} n_{2}-U_{1}$

Em que:

$\mathrm{n}_{1}=$ dimensão da menor amostra;

$\mathrm{n}_{2}=$ dimensão da maior amostra;

$\mathrm{R}_{1}=$ soma das ordenações da menor amostra.

\section{$3^{a}$ Etapa}

Uma vez que U é uma variável discreta, utilizou-se uma aproximação à normal nos casos de $\mathrm{n}_{1} \geq 10, \mathrm{n}_{2} \geq 10$, ou de $\mathrm{n}_{1}$ igual a 3 ou 4 e $\mathrm{n}_{2}>12$, sendo a expressão do teste dada pela Equação 3.

$Z=\frac{U-\frac{n_{1} n_{2}}{2}}{\sigma_{U}} \cap N(0 ; 1)$

Quando ocorreram empates entre elementos dos dois grupos e a dimensão dos empates não foi pequena, a expressão do desvio padrão foi dada pela Equação 4; para todas as outras situações, a expressão do desvio padrão foi dada pela Equação 5.

$\sigma_{U}=\sqrt{\frac{n_{1} n_{2}}{n(n-1)}\left(\frac{n^{3}-n}{12}-\sum_{j} \frac{t_{j}^{3}-t_{j}}{12}\right)}$

Em que:

$t_{j}=$ número de observações empatadas em cada grupo j

$\sigma_{U}=\sqrt{\frac{n_{1} n_{2}(n+1)}{12}}$

Depois que o valor de $\mathrm{Z}$ foi calculado, este foi comparado com o valor tabelado de uma distribuição normal para o teste bicaudal; o nível de significância admitido neste estudo foi de $5 \%$.

Se o valor de $Z$ estiver dentro da região de aceitação do teste, conclui-se que não existe diferença entre os grupos analisados; caso contrário, os grupos são estatisticamente diferentes, ou seja, os empresários identificam que uma variável encontrase em uma situação diferente das outras.

\section{RESULTADOS E DISCUSSÕES}

Diante das variáveis analisadas, as únicas percebidas em uma situação adequada referiram-se a disponibilidade de madeira, mão de obra com habilidades administrativas e disponibilidade de energia (Figura 3), pois estas se encontraram à direita do ponto de indiferença " 4 " da escala, exibindo um valor “ 5 ” de mediana. Embora essas variáveis tenham apresentado a mesma mediana, diferentemente de DM e DE, a variável MOHA apresentou uma assimetria, sugerindo, portanto, 


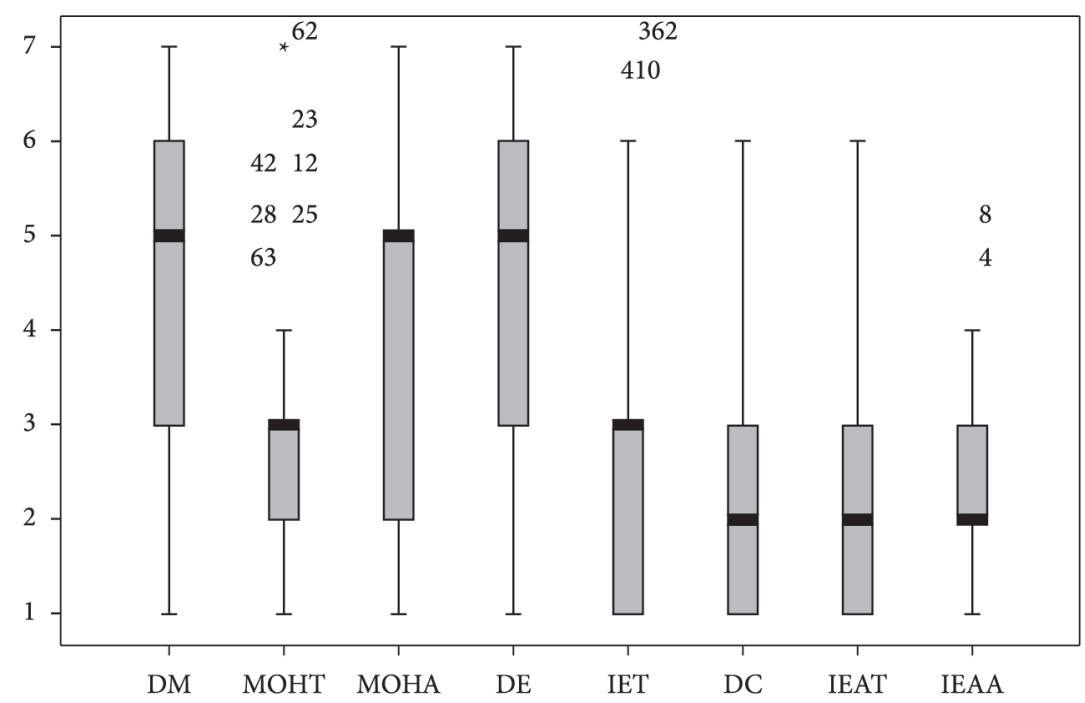

Figura 3. Resultados dos gráficos Boxplot para as variáveis analisadas.

Figure 3. Results graphics Boxplot for the variables analyzed.

uma pior situação. As demais variáveis apresentaram valores de mediana inferiores a " 4 ".

Os círculos e asteriscos da Figura 3 indicam os outliers, isto é, sete empresários apresentaram percepção muito diferente dos restantes para a variável MOHT, refletindo uma posição mais otimista a essa variável, comparativamente aos seus pares. De forma similar, identificaram-se quatro e dois outliers para as variáveis IET (infraestrutura para transporte) e IEAA (infraestrutura para aspectos administrativos), respectivamente (Figura 3 ).

A análise descritiva das variáveis pelo gráfico Boxplot indicou uma considerável discordância entre os empresários, haja vista a grande variabilidade das respostas expressas pelos seus valores mínimos e máximos, e as dispersões interquartis.

As variáveis que mostraram a menor variabilidade nas respostas foram MOHT e IEAA, não apresentando valores máximos dos seus gráficos Boxplot na condição de adequação. As respostas de todas as demais variáveis permearam praticamente toda a escala aplicada. A ampla dispersão das respostas reforça a necessidade da aplicação de um teste estatístico para a comparação das medianas.

Os resultados do teste de Mann-Whitney corroboraram os resultados sugeridos pelo gráfico Boxplot, indicando dois grupos de variáveis. O primeiro grupo destacou uma situação adequada para as variáveis referentes à disponibilidade de madeira, energia e mão de obra com habilidades administrativas.

No segundo grupo, reuniram-se os pontos críticos para o segmento de serrados, sendo: infraestrutura para transporte, disponibilidade de mão de obra com habilidades técnicas, disponibilidade de capital, infraestrutura para aspectos técnicos e infraestrutura para aspectos administrativos (Tabela 1).

Em geral, os resultados estão de acordo com o esperado, refletindo as solicitações do setor empresarial nacional em relação às precárias condições de infraestrutura no Brasil, o tão propagado "Custo Brasil". Também estiveram de acordo com trabalhos específicos para o segmento de serrados, ressaltando as vantagens do segmento no que tange à disponibilidade da sua principal matériaprima, a madeira.

Embora alguns analistas tenham apontado para uma possível escassez de madeira, em resposta ao forte aumento do preço da mesma entre 1999 e 2005 (Tuoto et al., 2004), Almeida et al. (2009) criticaram tais análises, demonstrando que essa alta de preço esteve relacionada a condições de curto prazo da demanda de madeira e não a questões estruturais de longo prazo da oferta; portanto, seria precipitado apontar a sua escassez no longo prazo. 
Tabela 1. Resultados do teste comparativo de grupos de Mann-Whitney.

Table 1. Results of the group's comparative test of Mann-Whitney.

\begin{tabular}{|cllc|}
\hline \multirow{2}{*}{ Sigla } & \multicolumn{1}{c}{ Variável } & \multicolumn{2}{c|}{ Grupos (mediana) } \\
\cline { 3 - 4 } & & $\mathbf{1}$ & $\mathbf{2}$ \\
\hline DM & Disponibilidade de madeira & 5 & \\
DE & Disponibilidade de energia & 5 & 3 \\
\hline MOHA & Mão de obra com habilidades administrativas & 5 & 3 \\
\hline IET & Infraestrutura para transporte & & 2 \\
\hline MOHT & Mão de obra com habilidades técnicas & 2 \\
\hline DC & Disponibilidade de capital & & 2 \\
\hline IEAT & Infraestrutura para aspectos técnicos & \\
\hline IEAA & Infraestrutura para aspectos administrativos & & \\
\hline
\end{tabular}

Em geral, o segmento de serrados não possui florestas e, em muitos casos, encontra-se em uma estrutura concentrada para a obtenção das mesmas, visto que a maior parte dos reflorestamentos encontra-se sob o domínio de grandes empresas de papel e celulose, e siderúrgicas (Almeida, 2006). Porém, ainda conforme o autor, dois fatores favorecem uma ampla disponibilidade de madeira para o segmento de serrados: primeiro, a alta produtividade das florestas plantadas no Brasil e, segundo, o temor pela falta de madeira das grandes empresas de base florestal.

As florestas plantadas no Brasil são as mais produtivas do mundo, alcançando uma produtividade, por exemplo, três vezes superior aos plantios dos EUA (Hoeflich, 2006), o que acaba beneficiando indiretamente o segmento de serrados. Já o alto custo de capital das grandes empresas de base florestal, associado ao longo prazo de maturação das florestas, obriga essas empresas a possuírem um planejamento conservador de seus reflorestamentos, minimizando ao máximo o risco de desabastecimento de madeira e aumentando a probabilidade de sobra da mesma (Almeida, 2006).

A percepção positiva quanto à disponibilidade de energia pode estar ligada a vários fatores, entre os quais as medidas do governo frente à ocorrência de um novo "apagão energético" e a menor relevância do custo de energia para produção de serrados, comparativamente ao custo de outros insumos.

Conforme IBGE (2011), enquanto os custos com matéria-prima e mão de obra respondem por, respectivamente, $56 \%$ e $34 \%$ dos custos totais industriais para produção de madeira serrada, a representatividade do custo de energia é de $10 \%$.

O "apagão energético" foi um racionamento forçado de $20 \%$ do consumo de energia em quase todo o País, entre os anos de 2001 e 2002. As suas causas combinaram a falta de planejamento e de investimento para o setor, sendo um dos principais responsáveis pela queda do PIB de $4 \%$ no primeiro trimestre do ano de 2001 para $-0,8 \%$ no primeiro trimestre de 2002 (Banderlin, 2004).

Porém, segundo o mesmo autor, diversas medidas foram tomadas pelos governos após o “apagão energético" de 2001, objetivando prover mecanismos que possam evitar problemas futuros de racionamento, como: investimentos preventivos na geração de energia elétrica; melhor planejamento do setor elétrico; expansão da interligação entre as regiões do País, e se o mercado não investir no sistema, o governo arcar com o investimento e/ou a captação de recursos. Além disso, muitos aspectos positivos puderam ser retirados do racionamento, entre os quais se destacaram: evolução da utilização de equipamentos com maior eficiência energética; criação de leis para incentivar tal utilização; incentivos para geração de fontes alternativas, principalmente da biomassa e eólica, e conscientização nacional em relação ao uso racional da energia elétrica.

$\mathrm{O}$ conflito entre os resultados referentes à disponibilidade de mão de obra com habilidades administrativas - situação favorável - e com habilidades técnicas - situação desfavorável é curioso, porém compreensível, dado que os respondentes do questionário, em geral, são os donos e administradores das empresas. 
Mostra-se curioso, porque, em geral, a economia brasileira tem sofrido uma escassez de mão de obra qualificada, administrativa e técnica, devido basicamente a dois fatores: a indisponibilidade de recursos humanos devido ao maior crescimento da economia nos últimos anos e à fraca qualificação da formação dos recursos humanos no País (Mascarenhas, 2011). Além disso, é curioso os empresários apontarem, por um lado, uma deficiência do Estado no treinamento dos recursos humanos, expresso pelas variáveis IEAT e IEAA (infraestrutura para aspectos técnicos e administrativos) e, por outro lado, uma disponibilidade favorável de mão de obra administrativa.

A percepção favorável do segmento de serrados quanto à disponibilidade de mão de obra administrativa pode ser parcialmente explicada pelo fato de que as empresas analisadas são pequenas ou médias. Estas, em sua maioria, não contam com a presença de recursos humanos qualificados administrativamente, provavelmente por entenderem que são profissionais caros e desnecessários, fenômeno comum ligado a pequenas empresas.

A percepção desfavorável para a infraestrutura pública para pesquisa, educação e treinamento doméstico para aspectos tecnológicos e administrativos é facilmente corroborado pela literatura. Para Lazzareschi (2010), as projeções de aumento do Produto Interno Bruto nos próximos anos desmascararam definitivamente a situação educacional do País, em alusão à falta de qualificação de mão de obra do país.

A autora critica a forma como é medida a taxa de desemprego do País e aponta que o Brasil ainda possui um número expressivo de desempregados, porém desqualificados. Para aqueles qualificados, é destacada a necessidade de a grande maioria, mesmo depois de formados, ter de se submeter a cursos de especialização oferecidos pelas próprias empresas contratantes, que se ressentem da formação deficiente.

Por fim, em relação à percepção desfavorável quanto à disponibilidade de crédito, dois aspectos devem ser levantados. O primeiro é que o crédito no Brasil é sabidamente caro, burocrático e que as linhas de crédito ainda são concentradas para grandes empresas. Segundo aspecto: o conhecimento leigo do gestor é outro complicador decisivo (Zolet \& Lozeckyi, 2007). Em seu trabalho, os autores apresentaram várias linhas e formas de crédito para micro e pequenas empresas.

\section{CONCLUSÕES}

Embora faça parte das reivindicações do setor empresarial apresentar um descontentamento incessante perante a atuação do governo, tal como o é para a classe trabalhadora, os resultados ensejam algumas conclusões.

A primeira conclusão é de que o setor empresarial minimiza os problemas de "apagão florestal e energético", teses amplamente alardeadas, e a segunda conclusão é de que reflete as deficiências na formação de recursos humanos para o segmento madeireiro, bem como corrobora com os tradicionais problemas nacionais de crédito e infraestrutura para transporte.

\section{STATUS DA SUBMISSÃO}

Recebido: 02 abr., 2012

Aceito: 07 ago., 2014

Publicado: 21 nov., 2014

\section{AUTOR(ES) PARA CORRESPONDÊNCIA}

\author{
Alexandre Nascimento de Almeida \\ Faculdade UnB de Planaltina - FUP, \\ Universidade de Brasília - UnB, Área \\ Universitária n. 1, Vila Nossa Senhora de Fátima, \\ CEP 73300-000, Brasília, DF, Brasil \\ e-mail: alexalmeida@unb.br
}

\section{REFERÊNCIAS}

Almeida AN. Estudo Econométrico da Demanda e Oferta de Madeira em Tora para o Processamento Mecânico no Estado do Paraná [Dissertação]. Curitiba: Setor de Ciências Agrárias, Universidade Federal do Paraná; 2006.

Almeida AN, Silva JCGL, Angelo H, Bittencourt AM, Nuñez BEC. Mercado paranaense de madeira em tora procedente de silvicultura entre 1999 e 2005. FLORESTA 2009; 39(4): 869-875. http://dx.doi.org/10.5380/ rf.v39i4.16322.

Almeida AN, Silva JCGL, Angelo H. Caracterização do ambiente de negócios para produção de madeira 
serrada no Brasil e no Canadá. FLORESTA 2011; 41(4): 751-764. http://dx.doi.org/10.5380/rf.v41i4.25354.

Andrioli, AI. O mito da competitividade. Revista Espaço Acadêmico 2003; 23. [cited 2010 Apr.]. Available from: http://www.espacoacademico.com.br/023/23and.htm.

Banderlin CEA. Os efeitos do racionamento de energia elétrica ocorridos no Brasil entre 2001 e 2002 com ênfase no consumo de energia elétrica [Dissertação]. São Paulo: Escola Politécnica, Universidade de São Paulo; 2004.

Furtado J. Mundialização, reestruturação e competitividade: a emergência de um novo regime econômico e as barreiras às economias periféricas. Novos Estudos CEBRAP 1992; 53: 23-46.

Hoeflich VA. Produção florestal sustentável de madeira para o mercado internacional: perspectivas e desafios. Embrapa florestas; 2006. [cited 2012 Mar.]. Available from: www.bioenergytrade.org/downloads/ hoeflichnovdec05.pdf.

Hoffmann R. Estatística para economistas. 4rd ed. São Paulo: Pioneira Thomson Learning; 2006.

Instituto Brasileiro de Geografia e Estatística - IBGE. Anuário Estatístico do Brasil. Rio de Janeiro: IBGE;
2011. [cited 2011 Mar.]. Available from: http://www. ibge.gov.br/home/.

Lazzareschi N. O apagão de mão de obra no Brasil. Ponto-e-vírgula 2010; 7: 192-199.

Mascarenhas AO. O que é o apagão da mão-de-obra e como se manifesta? 2011. [cited 2012 Mar.]. Available from: http://www.hsm.com.br/editorias/rh/o-que-e-oapagao-da-mao-de-obra-e-como-se-manifesta.

Salleroun L. Liberalismo e Socialismo. Tradução de João Benedicto Martins Ramos. São Paulo: Mundo Cultural; 1979.

Siegel S, Castellan NJ. Estatística não-paramétrica para as ciências do comportamento. 2. ed. Porto Alegre: Bookman; 2006. 448 p.

Tuoto M, Alves M, Scheffler L, Almeida E. O desequilíbrio do mercado de tora de Pinus no Brasil. STCP Informativo 2004; 7: 18-21.

Zolet JP, Lozeckyi J. Concessão de crédito para micro e pequena empresa. Revista Eletrônica Lato Sensu 2007; 2(1): 1-33. 